\title{
Prácticas clasificatorias desde la filosofía de la ciencia: entre metafísica y epistemología*
}

\author{
Classificatory Practices from Philosophy of Science: between \\ Metaphysics and Epistemology
}

Resumen: T. Reydon (2010) analiza el debate actual sobre los géneros naturales e identifica dos corrientes -denominadas "aproximación metafísica" y "aproximación epistemológica"- que interpretan y abordan un mismo problema de manera distinta. Después de examinar de forma crítica algunos aspectos problemáticos de la propuesta de Reydon se presenta un análisis alternativo, en donde se recogen y desarrollan aspectos de su propuesta introduciendo nuevos elementos a fin de salvar sus limitaciones. Defendemos que entender la discusión filosófica sobre los géneros naturales en los términos de este nuevo análisis facilita la comprensión y caracterización de muchos de sus aspectos más controvertidos.

Palabras clave: Géneros Naturales, Epistemología, Metafísica, Naturalidad, Membresía, Reydon.
ALBA AMILBURU MARTÍNEZ**

Recibido: 19/01/2017. Aceptado: 22/08/2017.

* La elaboración de este trabajo ha sido posible gracias a una beca predoctoral financiada por la Universidad del País Vasco (programa de ayudas FPI-2010). Agradezco a Cristian Saborido, Agustín Arrieta y Arantza Etxeberria sus útiles comentarios sobre borradores anteriores.

** Profesora en el Departamento de Lógica y Filosofía de la Ciencia de la Universidad del País Vasco. Líneas de investigación: prácticas clasificatorias, géneros naturales, la cuestión acerca de la definición de vida. Publicaciones recientes: "Metafísica naturalizada: géneros naturales en consonancia con la práctica científica". Teorema. Revista internacional de filosofía, 35 (2), 2016, 197-208, junto con C. Saborido y "Debate actual sobre los géneros naturales desde una perspectiva lockeana" en Ágora: papeles de filosofía, 35 (2), 2016, 133 155. Dirección de correo electrónico: alba.amilburu@ehu.eus. 


\section{Introducción}

La noción de género natural sirve como herramienta filosófica para reflexionar sobre los métodos clasificatorios, tanto en ciencia como más allá de la práctica científica. Tradicionalmente, se ha asumido que las categorizaciones científicas se correspondían con distinciones reales de los fenómenos que estas ciencias analizan. Consecuentemente, los científicos agruparían sus objetos de estudio en clases con propiedades objetivas compartidas, es decir, en clases que se diferenciarían unas de otras por sus propiedades "naturales". Así, la idea misma de clase o género natural puede ser interpretada como fundamento de las prácticas clasificatorias en ciencia.

Sin embargo, aún a pesar de este lugar central de la idea de género natural en filosofía de la ciencia, no hay un consenso acerca de qué y cómo es un género de este tipo. De hecho, ni siquiera está del todo claro cuál es la diferencia entre géneros naturales y géneros no-naturales.

En los últimos años, muchos autores han propuesto diferentes caracterizaciones de género natural. Por ejemplo, Dupré sugiere que los géneros naturales se distinguen del resto, básicamente, por razones pragmáticas en el ámbito de la investigación: "los géneros pueden distinguirse en función de propiedades incluyendo aquellas que son útiles desde el punto de vista económico o... de interés por razones teóricas” (1993, 113). En una línea muy diferente, Garvey (2007) afirma que los géneros naturales están “ahí fuera” en la naturaleza, puesto que no son imposiciones de nuestra propia conveniencia. Esta tesis es favorable a una posición realista en detrimento de una perspectiva convencionalista. En consonancia con esta última tesis, Ellis (2001) sostiene que los géneros naturales tienen esencias reales que fijan la identidad de tipo de las instancias que conforman estos géneros. Asimismo, Ellis asume que la estructura profunda del mundo está conformada por leyes y géneros naturales. LaPorte (2004), otro autor importante en esta discusión, afirma que los géneros naturales son naturales porque tienen valor explicativo, es decir, porque nos ayudan a explicar eventos y aspectos relacionados con las instancias que conforman el género natural en cuestión y es precisamente eso lo que los distingue de otro tipo de géneros. Por último, Boyd (1991, 1999) entiende que los géneros naturales son agrupaciones homeostáticas de propiedades generadas por mecanismos causales.

Como puede concluirse de esta notable divergencia de opiniones entre estos filósofos contemporáneos, hay un profundo desacuerdo de fondo en la forma de entender la idea de género natural, y estas divergencias quedan también de relieve a la hora de aplicar la idea de género natural a casos concretos. ¿Cómo justificar teóricamente que un término general, como, por ejemplo, "oro", "caballo", "azul", "objetos con carga positiva", es o no es un término de género natural? Con respecto a esta cuestión, encontramos diferentes posturas clásicas en filosofía. Una interpretación muy influyente de la idea de género natural ${ }^{1}$ es la de John Stuart Mill, quien afirmaba que: "un caballo es un Género porque las cosas que coinciden en poseer las propiedades por las cuales reconocemos a un caballo, también coinciden en un número elevado de propiedades distintas, como sabemos" (Mill 1843, 703-704). Mill

1 Sin embargo y de acuerdo con Hacking (1991), fue John Venn quien introdujo el término "género natural" en 1866, pues Mill hablaba de "géneros en la naturaleza". 
tenía como principal referencia el debate sobre las especies biológicas (Hacking 1991) y defendía, no obstante, que no todos los géneros pueden ser considerados como géneros en la naturaleza: "las cosas blancas, las cosas con carga negativa, las cosas con una masa de 1 $\mathrm{kg}$, no forman un género natural, porque intuitivamente no se puede afirmar que constituyan un Género" (Mill 1884). Esto contrasta con opiniones ulteriores más liberales sobre la idea de naturalidad, como la de Quine $(1969,49)$ para quién incluso los objetos con una carga positiva conformarían un género natural. A diferencia de lo que afirmaba Mill, Quine hace más flexible el criterio para distinguir géneros naturales de no-naturales porque asocia la naturalidad de estos géneros con la idea de similitud, lo que permite una interpretación más amplia de lo que constituye un género natural.

Este conjunto de consideraciones acerca de los géneros naturales muestra lo complicado que resulta hacerse con una idea medianamente clara de lo que son o no son estos géneros y de la idea de naturalidad que subyace a los mismos. Por esta razón, una propuesta de análisis meta-teórico - es decir, una interpretación del conjunto de las propuestas teóricas de género natural que conforman el debate actual- resulta de gran interés. La noción de género natural genera confusión y muchas dificultades a quien se propone comprenderla y en la actualidad determinar cuál es el estatus o carácter ontológico de los géneros naturales se ha mostrado por tanto una cuestión necesaria para avanzar en la reflexión filosófica. Así, elucidar el "horizonte implícito" que acompaña a la noción de género natural defendida en cada una de las propuestas teóricas o visiones de género natural que conforman el debate nos permitirá situar el debate contemporáneo y comprender por qué surgen tantas controversias y desencuentros entre los que participan en esta discusión filosófica. Para ello, primero nos fijaremos en el análisis del debate sobre los géneros naturales que propone Reydon, quien recientemente ha defendido la utilidad y el valor epistémico de la idea de género natural en filosofía de la ciencia (MacLeod y Reydon 2010; Reydon 2013; Ereshefsky y Reydon 2015). Este autor simpatiza con una concepción de género natural informada por las prácticas científicas. Lo que se podría entender como una "metafísica naturalizada" en lo que se refiere a la idea de género natural.

El objetivo principal de este trabajo es desarrollar y refinar el análisis propuesto por Reydon sobre el debate actual de los géneros naturales. Se trata de elaborar una propuesta que permita una mejor comprensión de esta discusión filosófica, capaz de clarificar los usos, las expectativas que genera el debate y el significado de los términos "género natural" y "naturalidad".

Con este objetivo en mente, en las secciones 2 y 3 se expone y analiza de forma crítica el análisis propuesto recientemente por Reydon. En estas secciones argumentaremos que la distinción entre una "aproximación metafísica" y una "aproximación epistemológica" de Reydon es problemática, y que lo que realmente distingue ambas líneas de trabajo es la idea de naturalidad que cada una de las corrientes principales que conforman el debate asume. Tras este análisis crítico, en la sección 4 se elabora una propuesta original de análisis meta-teórico del debate sobre los géneros naturales que, aunque basada parcialmente en la propuesta de Reydon, introduce nuevos elementos a fin de salvar sus limitaciones. En la sección 5, se responde a la cuestión acerca de la naturalidad de estos géneros. Por último, en la última sección se comentan algunas conclusiones. 


\section{El análisis de Reydon}

En un trabajo reciente, Reydon (2010) además de defender la importancia de la idea de género natural en filosofía de la ciencia, presenta un análisis meta-teórico del debate actual sobre los géneros naturales. En este análisis, el autor identifica dos corrientes o líneas de trabajo -que él mismo denomina "aproximación metafísica” y "aproximación epistemológica"- que interpretan y abordan un mismo problema filosófico de manera distinta.

Por un lado, está la "aproximación metafísica", en donde los géneros naturales se conciben como géneros reales que existen en la naturaleza, independientemente de la observación y del entendimiento humano. El origen de esta línea se remonta a Platón y Aristóteles, y los supuestos básicos que la acompañan son los siguientes: i) en la naturaleza, las cosas están agrupadas en géneros de acuerdo con su esencia real, ii) la identidad de género de cada clase de particular es única, iii) existe una clasificación única formada por géneros naturales que refleja el orden natural de las cosas en el mundo. El problema central que aborda esta línea de trabajo, según Reydon, es de carácter metafísico: partiendo del presupuesto que afirma que los géneros naturales están "ahí fuera" y que cada género natural está delimitado por su correspondiente esencia real, los teóricos de la "aproximación metafísica" (como p. ej., Ellis 2001, 2009 y Garvey 2007) se enfrentan a cuestiones tales como de qué manera se debe concebir y formular la idea de membresía natural y esencia real, o qué tipos de esencia real corresponden con los distintos tipos de géneros naturales que existen. Por último, Reydon considera que esta aproximación es deficiente porque de ella resulta una perspectiva de género natural demasiado limitada. De acuerdo con el autor, el proyecto teórico que se propone resolver esta línea de trabajo depende (requiere) de los resultados de la línea epistemológica y por esta razón, la "aproximación metafísica" trata de circunscribir esta dependencia mediante el establecimiento de criterios apriorísticos, como por ejemplo, el hecho de afirmar que todo género natural tiene una esencia real.

Por otro lado, Reydon identifica otra tradición o línea de trabajo de origen más reciente (más en consonancia con las ideas de Locke y, en general, con el empirismo británico del siglo XVII) a la que denomina "aproximación epistemológica”. En esta línea, los géneros naturales son considerados como agrupaciones de particulares realizadas por el ser humano con el propósito de que resulten útiles en determinados contextos. El problema aquí es de tipo epistemológico: la meta principal de los teóricos de la "aproximación epistemológica" (como por ejemplo, Boyd 1991) es comprender cómo y por qué ciertas agrupaciones de particulares resultan ser más apropiadas que otras para la predicción y la explicación. Según Reydon, esta "aproximación epistemológica" es también deficiente por ser demasiado liberal. Al contrario de lo que ocurría en la "aproximación metafísica", en esta línea no se determinan criterios claros para distinguir entre géneros naturales genuinos y géneros de otro tipo.

Después de hacer esta caracterización de las dos líneas de trabajo en el debate sobre los géneros naturales, Reydon defiende la siguiente idea: "puede parecer que hay dos líneas de pensamiento separadas sobre dos problemas distintos. [...] Pero esto sería malinterpretar la situación actual, ya que las dos líneas de pensamiento constituyen diferentes maneras de abordar el mismo problema" (Reydon 2010, 251). Para Reydon, ambas líneas de trabajo son aproximaciones a un único problema. Aunque no se específica en qué consiste este problema, Reydon deja claro que hay una conexión entre ambas líneas de trabajo. Las teorías 
exitosas de ambas aproximaciones deberían ser capaces de responder, en último término, a las cuestiones centrales que se plantean estas dos líneas. En palabras de Reydon:

Si los supuestos de la línea de trabajo metafísica -las cosas están agrupadas naturalmente en géneros y esos géneros existen de algún modo en el mundo- son correctos, cualquier aproximación metafísica de género natural lo suficientemente elaborada responderá inmediatamente las cuestiones epistemológicas: aquellos géneros que funcionan con éxito en el razonamiento humano son precisamente aquellos que existen en la naturaleza. En cambio, cualquier teoría aceptable que responda las cuestiones epistemológicas debe explicar en que sentido los géneros que funcionan en el razonamiento humano representan aspectos de la realidad (Reydon 2010, 251).

Por esta razón, Reydon concluye que la principal diferencia entre ambas aproximaciones está en la prioridad que cada una otorga a cuestiones de tipo metafísico o epistémico.

Resumiendo, Reydon defiende que las líneas de trabajo metafísica y epistemológica abordan un mismo problema y convergen al responder a las mismas cuestiones desde enfoques e intereses distintos. Esto es, ambas líneas son complementarias. De hecho, según el autor, una propuesta metafísica de género natural lo suficientemente aceptable debería responder satisfactoriamente las cuestiones que se plantea la línea epistemológica, y viceversa.

La propuesta de análisis de Reydon resulta de gran interés porque la idea de género natural es ambigua: como veremos hay un desacuerdo de fondo, es decir no hay consenso, sobre lo que cada una de las corrientes entiende por "natural". Por esta razón, antes de enfrentarnos a cuestiones como "qué son los géneros naturales", es conveniente abordar cuestiones del tipo "qué se entiende por "género natural"”. Así, un análisis meta-teórico como el que propone Reydon, es un paso previo necesario para comprender el debate actual, clarificar los usos y significado del término "género natural" y abordar de manera más efectiva la cuestión sobre qué son estos géneros.

\section{Crítica al análisis de Reydon}

Aún reconociendo la importancia del análisis de Reydon, se debe señalar que esta propuesta no está exenta de problemas. En particular, hay al menos dos razones principales por las que la distinción de Reydon entre una corriente metafísica y una epistemológica resulta inadecuada para dar cuenta del estado actual de esta discusión.

En primer lugar, esta distinción de Reydon reduce la dimensión metafísica de los géneros naturales al marco metafísico esencialista ${ }^{2}$. En consecuencia, la línea de trabajo que Reydon denomina "metafísica" sería en realidad una línea "esencialista", lo que obvia la dimensión metafísica que también subyace a los enfoques no-esencialistas, como por ejemplo la

2 De acuerdo con la concepción esencialista de género natural los géneros naturales tendrían esencias. Estas esencias definen la identidad de tipo de las instancias que conforman el género -sirven a modo de criterio de membresía- y explican sus propiedades características. A su vez a cada género natural le corresponde una única esencia. Se considera que la idea de esencia es una idea de carácter metafísico. De ahí la denominación "marco metafísico esencialista". Sin embargo, como se defenderá a lo largo de este trabajo, esta dimensión metafísica (relacionada con la idea de género natural) no se agota con la concepción esencialista. 
propuesta de Boyd (1999) que entiende los géneros como agrupaciones homeostáticas de propiedades, propuesta teórica que introduciremos en la siguiente sección.

En segundo lugar, no es posible distinguir entre aproximaciones netamente metafísicas o epistemológicas, pues son aspectos interdependientes en gran medida. Los supuestos metafísicos asociados con las propuestas teóricas de género natural -en particular, en lo referente a las formulaciones de la condición de membresía- acarrean importantes consecuencias epistémicas. De hecho, la formulación (en términos metafísicos) de un criterio de membresía (criterio clasificatorio) añade dirección, orden y significado a proyectos de investigación particulares. Asimismo, como se muestra en la siguiente sección, algunas teorías de género natural combinan aspectos de tipo metafísico y epistémico. Por lo que una distinción dicotómica de estas características resulta algo forzada, si nuestro propósito consiste en comprender y reflejar la configuración actual del debate sobre estos géneros.

Por tanto, las tesis principales que se defienden en este trabajo son las siguientes: (i) la distinción entre una "aproximación metafísica" y una "aproximación epistemológica" de Reydon es problemática y no refleja de manera adecuada la configuración actual del debate. (ii) Lo que realmente distingue ambas líneas de trabajo no es una cuestión de simple prioridad entre cuestiones metafísicas o epistemológicas, sino la idea de naturalidad que cada una de estas líneas asume.

De este modo, en este artículo nos proponemos desarrollar y refinar la propuesta programática ofrecida por Reydon. Así, en lugar de entender el debate entre las diferentes propuestas teóricas sobre los géneros naturales como una discusión dicotómica entre una "aproximación metafísica" y una "aproximación epistemológica", tal y como propone Reydon, el análisis alternativo que se desarrolla en la próxima sección propone reinterpretar el debate en términos de una "concepción unitaria", otra "integradora" y una última que denominaremos "promiscua".

\section{Un análisis alternativo: concepciones unitaria, integradora y promiscua}

Entre las aproximaciones realistas de género natural que conforman este debate podemos distinguir tres visiones que denominaremos "unitaria", "integradora" y "promiscua". En líneas generales, los autores afines a la primera visión, la unitaria, defienden que hay un único modo de clasificar las cosas en géneros de manera natural, mientras que los de la concepción integradora sostienen que hay un modo integrado, más sofisticado, que combina varias maneras de "cortar la naturaleza por sus junturas", esto es, de agrupar naturalmente las cosas en géneros, ya que según esta concepción los géneros naturales apelan a una identidad de tipo que es, como veremos, realizable de manera múltiple. Por último, de acuerdo con la concepción promiscua, se renuncia a una idea de "membresía natural" establecida apriorísticamente y se defiende que existen muchos modos naturales, o adecuados, de agrupar las cosas en géneros que dependen en gran medida de los intereses teóricos que motivan la investigación.

Más concretamente, propuestas tales como la de Ellis (2001, 2009), Bird (2010a, 2010b, 2010c), Garvey (2007), Devitt (2008), Lowe (2006), Kripke (1972) y Putnam (1975) pueden ser agrupadas bajo la etiqueta "concepción unitaria de género natural" porque ofrecen una única respuesta a cómo agrupar los fenómenos (entidades, objetos, procesos, etc.) en géne- 
ros naturales. Así, de esta concepción resulta una sola respuesta a la cuestión ¿qué tipo de cosa es un género natural? En esta línea, la naturalidad de estos géneros apela a la idea de "membresía natural" que subyace a esta concepción, la cual es entendida como "esencia" en un sentido aristotélico 3 . De hecho, este conjunto de propuestas unitarias está íntimamente ligado a la perspectiva esencialista, lo que significa que, por un lado, se considera que la esencia es condición necesaria y suficiente para determinar la identidad de tipo, delimitando a su vez el género, y por otro, se afirma que la esencia explicaría la presencia de las propiedades típicas que encontramos en los miembros del género en cuestión ${ }^{4}$. De ahí que todo aquel género que cuente con una condición de membresía que se ajusta a esta idea de "esencia" sea considerado como un género natural genuino. En resumen, si nos preguntamos cómo podemos decidir cuál es el criterio de membresía apropiado para agrupar y dividir las cosas en géneros -siempre de un modo natural- la respuesta, de acuerdo con esa concepción unitaria, la encontramos en la idea de "esencia". Esto es, la "esencia" es el criterio que nos orienta a la hora de identificar géneros que son naturales.

Por otro lado, en este mismo debate también hallamos propuestas teóricas de género natural que designaremos "concepciones integradoras". Las propuestas de Boyd (1991, 1999), Wilson (1999), Wilson, Brigandt y Barker (2007), Khalidi (2013) y Slater (2014) pueden situarse en esta categoría. Consideramos que todas ellas son "integradoras" porque tratan de ofrecer una explicación -un marco teórico unificado, aunque flexible- que dé respuesta a la cuestión sobre la naturaleza de los géneros naturales. En este caso, y en contraste con la concepción unitaria, la idea de "membresía natural” explica sólo parcialmente en qué consiste la naturaleza de los géneros naturales porque esta naturaleza también está ligada a las "demandas de acomodación” (cfr. Boyd 1999) o, dicho de un modo distinto, a los intereses epistémicos y pragmáticos relativos a las prácticas clasificatorias de las distintas disciplinas.

De este modo, estas propuestas teóricas integradoras se caracterizan por flexibilizar la idea de membresía natural o "esencia" que encontramos en la concepción unitaria. Esto es, aquí la membresía natural no apela a una idea de esencia en el sentido clásico aristotélico, sino más bien a una condición de membresía de carácter más disyuntivo. Esto nos conduce a entender la identidad de tipo, asociada a cada género, como algo que es realizable de manera múltiple. Por ejemplo, según Boyd los géneros naturales -entendidos como agrupaciones homeostáticas de propiedades (AHP)- están determinados por una agrupación o familia de propiedades, presentes en las instancias, que tienden a darse juntas. De hecho, la generación o aparición conjunta de propiedades es el resultado de una suerte de homeostasis, es decir, la presencia de algunas propiedades tiende a favorecer (bajo circunstancias apropiadas) la presencia de otras y se forma, así, una familia de propiedades que muestra cierta estabilidad. Esto ocurre regularmente debido a que hay mecanismos causales que hacen que la aparición de unas propiedades refuerce la aparición de otras. Así pues, los miembros de estos géneros naturales exhiben combinaciones de propiedades que son el resultado de la acción de

3 Por ejemplo, de acuerdo con la concepción unitaria, la propiedad esencial del oro es su número atómico, 79. El número de protones, que coincide con el número de electrones, determina la identidad de tipo y explica, a su vez, las propiedades físico-químicas del oro.

4 Los casos de Bird y Putnam son peculiares. Putnam flexibiliza su idea de esencia con el paso del tiempo, asociándola con la idea de similitud, mientras que Bird considera que la esencia de los géneros naturales es una propiedad necesaria pero no suficiente (Bird y Tobin 2017). 
mecanismos homeostáticos que pueden ser intrínsecos o extrínsecos (es decir, relacionales). Autores como Boyd, Wilson, Barker y Brigandt consideran que muchos géneros en biología, como las especies biológicas, son ejemplos de géneros naturales entendidos como AHP.

Por tanto, se puede apreciar que al igual que en el caso de la concepción esencialista unitaria de género natural Boyd asocia la idea de género natural con una dimensión causal. No obstante, Boyd reinterpreta esta dimensión causal (Wilson et al. 2007, 197) y re-formula los aspectos metafísicos que asociamos con los géneros naturales, ofreciendo una caracterización de estos géneros sin recurrir a propiedades esenciales (necesarias, suficientes y que explican las propiedades típicas de los miembros que conforman el género natural en cuestión) que definan la identidad de tipo, en el sentido tradicional. Boyd, al igual que sus seguidores, ofrece una solución disyuntiva al problema de la membresía del género natural, esto es, se trataría de un criterio de pertenencia (o "esencia") disyuntivo dentro de los límites que marca (en el marco teórico que adopta Boyd) la agrupación de propiedades causada por un mecanismo homeostático (Borghini y Casetta 2012). Esto implica que distintas combinaciones de propiedades (de la misma familia de propiedades) puedan apuntar a la misma identidad de tipo, única para cada género natural.

Por tanto, en esta concepción integradora se plantea que hay un modo que combina, a su vez, varios modos de agrupar las cosas de manera natural en géneros. Esto es, si nos preguntamos cómo podemos decidir cuál es el criterio de membresía apropiado para determinar si se trata o no de un género natural la respuesta apunta a una idea de "esencia" disyuntiva y más flexible, así como a las demandas de acomodación relativas a las distintas disciplinas científicas. Recordemos que en la propuesta integradora, a diferencia de lo que ocurre en la concepción unitaria, la teoría sobre la membresía natural de los géneros naturales está condicionada y es en parte relativa a las distintas demandas de acomodación. En la concepción unitaria la idea de "membresía natural" coincide con la idea de esencia tradicional o aristotélica y esta es independiente de los intereses epistémico-pragmáticos de la investigación.

Por último, denominaremos las propuestas de género natural de Dupré (1993, 2002), Hacking (1991) y Magnus (2012) “concepciones promiscuas”, pues en contraste con lo que ocurre en las dos concepciones anteriores, estos autores consideran que la naturaleza de los géneros naturales debe entenderse en un sentido pluralista y heterogéneo. Así, en la opinión de estos autores existirían múltiples respuestas para abordar la cuestión "qué tipo de cosa es un género natural", ya que la naturalidad de estos géneros no apelaría a una idea concreta de "membresía natural" expresada en términos generales (para todo caso), esto es, en términos de esencia tradicional o criterio disyuntivo. En consecuencia, no asumen compromiso alguno con una determinada idea de membresía natural como ocurre en los dos casos anteriores. No teorizan sobre una idea única de "membresía natural".

En síntesis, en la concepción promiscua consideran que hay muchas maneras de agrupar los fenómenos en géneros naturales. De modo que a la hora de decidir cuál es el criterio de membresía apropiado para cada caso estos autores renuncian a un criterio basado en la idea de membresía natural y se fijan en las demandas de acomodación y en los aspectos epistémico-pragmáticos propios de la prácticas clasificatorias en ciencia.

Por lo tanto y volviendo al objetivo que nos ocupa en este trabajo, en contraste con lo que afirmaba Reydon, atendiendo a este nuevo análisis meta-teórico, no parece probable que una propuesta "metafísico-esencialista" de género natural lo suficientemente elaborada, 
como afirma Reydon, pueda responder las cuestiones que se plantean en la otra línea de trabajo. Esto se debe, básicamente, a que la primera línea se compromete con supuestos metafísicos de tipo esencialista que la segunda y la tercera línea rechazan y por tanto los géneros naturales que cada una de las líneas reconoce como tales no son aceptados como naturales por las otras líneas. Dicho de otro modo, las clases de los géneros naturales (el conjunto de géneros) de las distintas concepciones no son coextensivas. Así y de acuerdo con Hacking $(2007,204)$ creemos que muchas de las teorías de género natural que encontramos en el debate actual son incompatibles, al menos en gran medida. Como ya se ha adelantado, y se desarrolla de manera más extensa en la siguiente sección, esta incompatibilidad tiene relación con la idea de membresía natural que subyace a cada una de las concepciones de género natural, pues tiene implicaciones y establece límites en cada una de las concepciones. Límites que no serán compartidos.

\section{5. ¿En qué sentido son estos géneros "naturales"?}

Una de las principales virtudes de esta nueva propuesta de análisis es que muestra que, en este debate, los géneros pueden ser naturales, al menos, en tres sentidos distintos. En primer lugar, los géneros son naturales en el sentido esencialista. Los géneros naturales tienen esencias reales ${ }^{5}$ y son naturales en función de su esencia real, es decir, siempre y cuando el criterio de membresía del género se ajuste a la definición propuesta en este marco esencialista. Los teóricos y seguidores de esta línea de trabajo teorizan sobre la formulación apropiada de esencia real. Cabe añadir que de acuerdo con la concepción unitaria, la naturalidad es una cuestión de todo-o-nada que se expresa en términos de "membresía natural" y que determina claramente si un género es o no natural, excluyendo la gradualidad. Los géneros naturales son independientes de nosotros y están "ahí fuera" en la naturaleza, nuestra labor consiste en reconocerlos e identificarlos.

De acuerdo con la concepción integradora, los géneros naturales son fruto de la interacción entre los intereses, las prácticas humanas y las estructuras causales que son independientes de estas prácticas (Boyd 1999, 2010; Keller et al. 2003). Aun así, como ya se ha mostrado en esta concepción es posible identificar una idea de "membresía natural" que sirve como fundamento de la idea de naturalidad y que, en principio, puede acomodar aspectos relativos a los intereses humanos. Por tanto, en esta concepción la idea de naturalidad es en parte relativa a las distintas disciplinas, ya que cada disciplina cuenta con diferentes familias de propósitos inferenciales y prácticas, y también contempla la idea de "membresía natural"'. Esto significa que en la idea de naturalidad de esta concepción se reconocen y recogen aspectos de tipo metafísico, así como de tipo epistemológico, algo que contrasta y no encaja bien con lo que defiende Reydon cuando distingue entre aproximaciones metafísicas y epistémicas. La concepción integradora es una posición intermedia que se sitúa entre la concepción unitaria y la promiscua.

5 En el sentido que determina Locke (1975). Se tiene en cuenta la distinción entre esencia real y nominal. La idea de esencia real lockeana coincide con la idea de esencia aristotélica (Ayers 1981, 256).

6 Una formulación de la "estructura causal independiente de nuestras prácticas" o de la dimensión causal asociada con los géneros naturales. 
En último lugar, la idea de naturalidad que subyace a la concepción promiscua no se expresa en términos de membresía natural sino que tiene relación con la capacidad predictiva y explicativa del género y con el interés teórico que éste despierta. Esto es, los géneros naturales son útiles para alcanzar propósitos teóricos. Un género es natural cuando se ajusta a los requerimientos epistémicos y teóricos, relativos al contexto de la investigación. En consecuencia, la idea de "normatividad natural", asociada con idea de naturalidad, que resulta de esta línea de trabajo es mucho más liberal que en las anteriores concepciones porque le falta esa fundamentación expresada en términos metafísicos.

Por último, cabe señalar que los compromisos metafísicos que se asumen en este debate también varían de una concepción a otra. Bird (2010a) desarrolla la cuestión acerca de la dimensión metafísica propia de los géneros naturales al considerar el grado de compromiso metafísico que asumen las distintas propuestas teóricas de género natural en el debate. De acuerdo con Bird, se pueden identificar varios grados de compromiso metafísico.

El primer grado de compromiso metafísico se formula en relación con la tesis que afirma que hay distinciones naturales genuinas entre las cosas, es decir, se refiere a las diferencias y similitudes genuinas que hay entre las cosas (en lo que se refiere por ejemplo al aspecto físico). La visión que asume esta tesis Bird la denomina "weak realism" (realismo débil). De acuerdo con el realismo débil, según Bird, hay divisiones naturales entre las cosas y nuestras actuales clasificaciones tienen éxito (o fallan) al identificar dichas divisiones.

El segundo grado de compromiso metafísico está relacionado con la cuestión acerca de la idea de membresía. Bird se pregunta si es posible formular una idea de membresía sobre la base de estas divisiones y distinciones naturales genuinas. No es lo mismo una agrupación de cosas que refleja similitudes y diferencias genuinas entre cosas o un género que refleja una demarcación natural entre géneros de cosas. El criterio acerca de la membresía natural es un criterio más exigente, que requiere un mayor grado de abstracción, en comparación con la idea de distinción natural.

Después de esta serie de consideraciones, se puede apreciar que la primera corriente -la "aproximación unitaria"- se compromete claramente con ambas tesis metafísicas, formulando el segundo aspecto metafísico en términos esencialistas, mientras que la visión promiscua, se compromete claramente con la primera tesis metafísica pero deja abierta la cuestión acerca del compromiso con la segunda tesis metafísica. De hecho, en esta línea, la formulación metafísica de la idea de membresía queda condicionada a una función compleja; a saber, la contribución epistémica del género en función del interés y del contexto particular. La interpretación de la dimensión realista que acompaña a la idea de género natural es distinta en cada caso y esto -además de las diferentes acepciones de la idea de naturalidadexplica en parte la confusión y controversia que se genera en el debate.

\section{Conclusiones}

A modo de conclusión la crítica que se presenta en este trabajo al análisis de Reydon se basa en tres puntos principales. En primer lugar, la distinción entre una "aproximación metafísica" y una "aproximación epistemológica" de Reydon no es adecuada para interpretar la geografía de este debate. Reydon reduce la dimensión metafísica de los géneros naturales al marco metafísico esencialista, con lo que "metafísico" y "esencialista" vienen a significar 
lo mismo. Esta "sinonimia" no es correcta, si reparamos en la concepción integradora, la cual reformula la dimensión metafísica asociada con los géneros naturales, y se compromete con una idea distinta de membresía natural.

Segundo, no puede establecerse una distinción tajante entre una aproximación metafísica y una epistemológica, ya que la concepción integradora no encaja dentro del análisis de Reydon (o encaja en ambas aproximaciones a la vez) al combinar ambos tipos de aspectos, tanto metafísicos como epistémicos. Como se ha explicado en las secciones anteriores la aproximación integradora reformula los aspectos metafísicos (y causales) asociados con los géneros naturales ofreciendo una respuesta alternativa a la cuestión sobre la naturalidad.

En tercer lugar, lo que realmente distingue a las distintas teorías acerca de los géneros naturales no es una cuestión de simple prioridad entre cuestiones metafísicas o epistemológicas, como defiende Reydon, sino la idea de naturalidad que cada una de estas teorías adopta. En contraste con el análisis de Reydon, la propuesta de análisis presentada en este trabajo explica la razón de la ambigüedad en el uso del término "género natural". Esta ambigüedad se debe a las distintas ideas de naturalidad que subyacen a las líneas de trabajo, y ésta es la razón de la tensión, confusión y dificultades que se generan entre los autores que participan en esta discusión.

Partiendo de estos tres puntos, en este artículo se ha presentado y defendido, con respecto al debate sobre los géneros naturales, un análisis meta-teórico alternativo que supone un desarrollo y refinamiento de la propuesta programática ofrecida por Reydon. Este nuevo análisis entiende el estado actual del debate filosófico en términos de una discusión entre una línea de trabajo unitaria, integradora y otra promiscua. Creemos que esta propuesta supone una herramienta útil para comprender e interpretar las peculiaridades que presentan las distintas propuestas teóricas de género natural y las expectativas que genera este debate en torno a la reflexión sobre los métodos, las prácticas clasificatorias y la adecuación de los criterios clasificatorios empleados, tanto en ciencia como más allá del ámbito científico.

\section{Referencias}

Ayers, M. (1981). Locke versus Aristotle on Natural Kinds. Journal of Philosophy, 78, pp. 247-72.

Bird, A. "The Metaphysics of Natural Kinds", recuperado de http://eis.bris.ac.uk/ plajb/ research/inprogress/Metaphysics_Natural_Kinds.pdf.

Bird, (2010), «A Posteriori Knowledge of Natural Kind Essences: A Defense», Philosophical Topics, Vol. 35, pp. 293-312.

Bird, (2010), «Discovering the Essences of Natural Kinds», en H. Beebee, and N. SabbartonLeary (eds.), The Semantics and Metaphysics of Natural Kinds, Routledge, pp. 125-136.

Bird, Alexander and Tobin, Emma, "Natural Kinds", The Stanford Encyclopedia of Philosophy (Spring 2017 Edition), Edward N. Zalta (ed.), URL $=<$ https://plato.stanford.edu/ archives/spr2017/entries/natural-kinds/>.

Borghini, A., y Casetta, E. (2012), «Quel che resta dei generi naturali», Rivista di estetica, Vol. 49, pp. 247-273.

Boyd, R.N. (1999), «Kinds, Complexity and Multiple Realization», Philosophical Studies, 95, pp. 67-98. 
Boyd, R.N. (1991), «Realism, anti-foundationalism and the enthusiasm for natural kinds», Philosophical Studies, 61, pp. 127-148.

Boyd, R.N. (1999), «Homeostasis, Species, and Higher Taxa», en R. Wilson (eds.), Species: New Interdisciplinary Essays. Cambridge: MIT Press, pp. 141-185.

Cooper, R. (2005), Classifying madness: A philosophical examination of the diagnostic and statistical manual of mental disorders. Springer,.

Devitt, M. (2008), «Resurrecting Biological Essentialism», Philosophy of Science, 75, $\mathrm{n}^{\circ}$ 3, pp. 344-382.

Dupré, J. (1993), The Disorder of Things: Metaphysical Foundations of the Disunity of Science. Harvard: Harvard University Press.

Dupré, J. (1999), «Are Whales Fish?», in D.L. MEDIN \& S. ATRAN, (eds), Folkbiology, MIT Press, pp. 461-476.

Dupré, J. (2002), Humans and Other Animals, Oxford: Oxford University Press.

Ellis, B. D. (2001), Scientific essentialism. Cambridge: Cambridge University Press.

Ellis, B. D. (2009), The Metaphysics of Scientific Realism, Chesham: Acumen.

Ereshefsky, M., \& Reydon, T. A. (2015). Scientific kinds. Philosophical Studies, 172 (4), 969-986.

Garvey, B. (2007), Philosophy of biology. Stocksfield: Acumen.

Goodman, N. (1978), Ways of Worldmaking. Indianapolis: Hackett.

Hacking, I. (1991), «A Tradition of Natural Kinds», Philosophical Studies, Vol. 61, pp. $109-126$.

Hacking, I. (2007), «Natural kinds: Rosy Dawn, Scholastic Twilight». Royal Institute of Philosophy Supplement, Vol. 82, $\mathrm{n}^{\circ}$ 61, pp. 203-239.

Keller, R. A., Boyd, R. N., \& Wheeler, D. Q. (2003), «The illogical basis of phylogenetic nomenclature». The Botanical Review, Vol. 69, n 1, 93-110.

Khalidi, M. A. (2013), Natural Categories and Human Kinds. Classification in the Natural and Social Sciences. Cambridge: Cambridge University Press.

Laporte, J. (2004): Natural Kinds and Conceptual Change. Cambridge: Cambridge University Press.

Locke, J. (1975): An Essay Concerning Human Understanding. En Peter H. Nidditch (ed.). New York: Oxford University Press. Traducción de E. O’Gorman. Ensayo sobre el entendimiento humano. México: Fondo de Cultura Económica.

Lowe, E. J. (2006): The Four-Category Ontology: A Metaphysical Foundation for Natural Science, Oxford: Clarendon Press.

Macleod, M. (2010): «The Epistemology-only Approach to Natural Kinds: a Reply to Thomas Reydon», en F. STADLER (ed.) The Present Situation in the Philosophy of Science. The Philosophy of Science in a European Perspective, Vol. 1, pp. 189-194.

MacLeod, M., \& Reydon, T. A. (2013). Natural kinds in philosophy and in the life sciences: Scholastic twilight or new dawn?. Biological Theory, 7 (2), 89-99.

Magnus P. D. (2012): Scientific Enquiry and Natural Kinds: From Planets to Mallards, Basingstoke, Hampshire: Palgrave Macmillan.

Mill, J. S. (1884): A System of Logic. London: Longman.

Okasha, S. (2002): «Darwinian Metaphysics: Species and the Question of Essentialism», in Synthese, 131, pp. 191-213. 
Quine, W. O. (1969): Ontological Relativity and Other Essays. New York: Columbia University Press.

Reydon, T. (2010): «Natural Kind Theory as a Tool for Philosophers of Science» En M. Suárez, M. Dorato, \& M. Rédei (eds.), EPSA, Springer, pp. 245-254.

Russell, B. (1948): Human knowledge: its scope and limits. New York: Simon and Schuster.

Slater, M. (2014): «Natural Kindness», The British Journal for The Philosophy of Science, doi: 10.1093/bjps/axt033

Strawson, P. F. (1959): Individuals, London: Methuen, p.169.

Wilson, R. A. (1999): «Realism, essence, and kind: resucitating species essentialism», en Species: New Interdisciplinary Essays, Cambridge: MIT Press, pp. 187-207.

Wilson, R., Barker, M. J., \& Brigandt, I. (2007): «When traditional essentialism fails: biological natural kinds», Philosophical Topics, Vol. 35, n 1, pp. 189-215. 
